\title{
The $27^{\circ}$ World Congress on Controversies in Obstetrics, Gynecology \& Infertility (COGI) \\ Paris, France - November 21 - 23, 2019
}

\section{OVARIAN AND ENDOMETRIUM PLATELET-DERIVATED GROWTH FACTOR (PDGF) THERAPY IN REPRODUCTIVE MEDICINE}

Prof G. Menaldo, A. Giovannelli, C. Conti

Centro Clinico San Carlo di Torino, Unità di Procreazione Assistita, Torino, Italy

PROBLEM STATEMENT: Platelet rich plasma (PRP) has been previously used with some success in various non-reproductive medical contexts because its relevant tissue regeneration processes. One step further was the use of Platelet-Derived Growth Factor (PDGF) because it's not necessary cells translation but growth factor translation. In this study we extended PDGF application to reproductive medicine to overcome problem of patients with low (or absent) ovarian reserve and patients who cannot obtain adequate endometrial thickness for successful embryo transfer.

In this study we evaluated the effectiveness of ovarian PDGF injection, in order to rejuvenate and reactivate ovaries with the ultimate goal of indicating a possible correlation between this mode of treatment and reproductive effect in women with diminished ovarian reserve as determined by at least one prior IVF cycle canceled for poor follicular recruitment response.

PDGF application in endometrium has also been associated with increased of thickness and progesterone receptor activity. Progesterone receptors are the main actors that help maintain a thick and healthy endometrial lining, which in turn, helps with embryo implantation.

METHODS: We have started offering ovarian and endometrium PDGF applications for 122 patients between 32 and 47 years old with: IVF failures, poor oocyte yield and POF (Premature Ovarian Failure) and patients who cannot obtain an adequate endometrial thickness. Ovarian infusion of PDGF is programmed in 8-10 day of menstrual cycle while endometrial PDGF application is administered approximately 48-96 $\mathrm{h}$ before embryotransfer.

PDGF was prepared from autologous blood using RegenACR kit according to manufacturer's instructions. After controlling platelet degranulation at microscope, $1 \mathrm{cc}$ of pure PDGF was infused on ovaries using a thin needle under transvaginal ultrasound guidance and/or infused on the endometrium using Gynetics catheter.

RESULTS: To verify action of this treatment we attach importance to presence of dominant follicle in 8-10 day of next cycle; we also check the hormonal profile of FSH and E2 in 3rd day of menstrual cycle; after application of PDGF, the endometrial thickness was satisfactory in all patients ( $>8 \mathrm{~mm})$, with endometrial three-layer pattern.

The results of PDGF treatment in ovary and endometrium are promising because $36 \%$ of these patients with previous IVF failures achieved pregnancy ( 41 women).

CONCLUSION: In our experience, PDGF treatments seems to have an important regenerative function of reproductive system. These results translates into several pregnancies, registered in all women with previous IVF failures who have never had a pregnancy before.
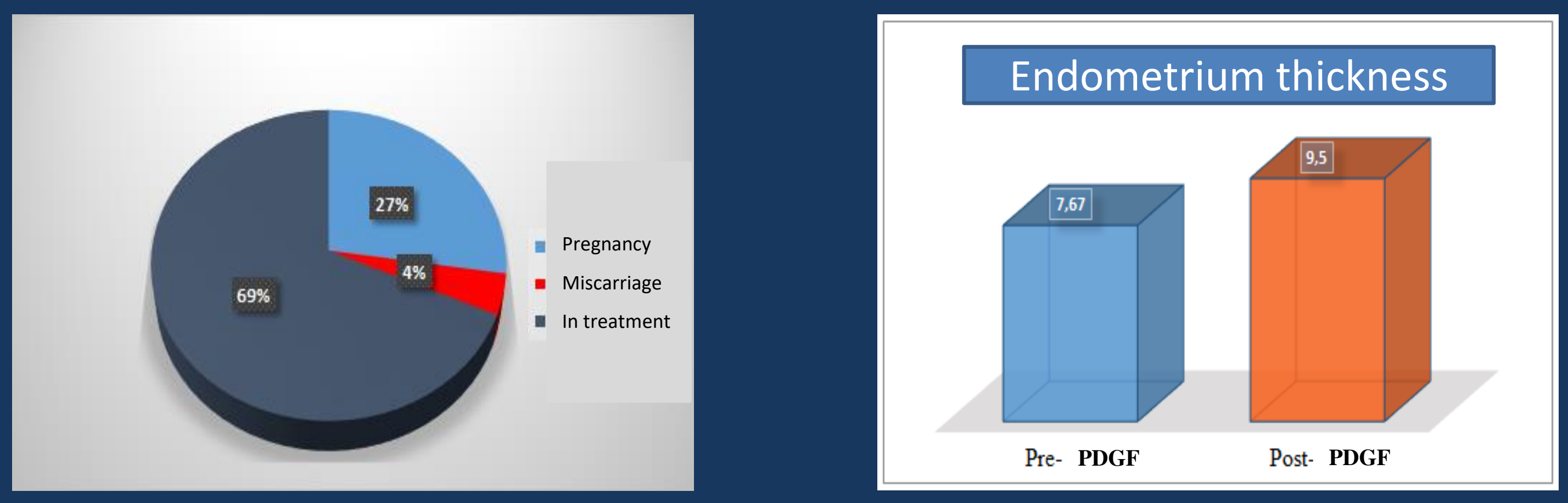